\title{
Manuscript Formatting, Delayed Peer-reviews, and Overemphasizing the Impact Factor: Can Something be Done?
}

\author{
Vijay Kothari \\ Institute of Science, Nirma University, Ahmedabad, India. \\ vijay.kothari@nirmauni.ac.in; vijay23112004@yahoo.co.in
}

Keywords: Peer-review, Impact Factor, Pre-print

Academicians and researchers do experiments, and then communicate their findings to a journal found to be compatible by them, for publishing their findings. Current scenario of the publication process is that each of the journals have their own formatting requirements with respect to font size, reference style, figure resolution, table and chart type, etc. Many of them also pose limits on maximum number of figures/ tables which can be allowed in a single article. This compels the academicians/ research fellows to spend quality time on manuscript formatting rather than doing actual science in lab. As it happens in nearly half of the cases, papers do get rejected, and needs to be resubmitted to another journal; which means another cycle of formatting and reformatting, which of course is not guaranteed to be accepted for publication.

Let us just think, whether this formatting really makes any difference to the scientific quality of the data reported in a particular study? Most of us will agree, that the answer is a 'no'. Then why so much emphasis on formatting the manuscripts strictly as per certain guidelines? Taken together for the global research community, a lot many human hours are sacrificed on this mundane formatting exercise, which otherwise can be spent in lab or library in a much better way. It seems wise to propose a uniform formatting style for all scientific manuscripts (i.e. one style for all research papers, and another one for all review papers); even better may be not to lay down any formatting requirements to the extent that scientific quality and readability of data does not get affected.

Even after paying enough attention to the formatting demands of the journals, researchers are not guaranteed to receive reviewer's comments on their manuscript within a reasonable time period. All reviewers need to be sensitive towards the undue delay. Traditionally reviewing has remained a job being done on voluntary basis, and no rewards (except appreciation and acknowledgement) are usually offered. One of the reasonable ways to expedite the review process seems to be paying some honorarium to the reviewers and editors for their time spent in reviewing and editing activities. Publons [https://publons.com/home/] is a good initiative for showcasing the reviewing and editorial contributions of experts to their respective fields. But more needs to be done to ensure that good quality of peer-review can be performed on majority of manuscripts without too much delay. Particularly when open-access publishing is gaining ground, wherein authors are required to pay for publishing their manuscript, publishers can surely afford to give some share of their profit to the reviewers, who for sure play an essential role in the whole publication process. If not cash rewards always, then at least full (and not partial) waiver on 'page charges'/ 'open access fee' must be offered to the reviewers, when they submit their own manuscript to the journal for which they acted as reviewer. Concepts like 'Post-publication review' and 'open review' have been floated, but overall situation has not changed much. Platforms like bioRxiv [http://www.biorxiv.org/] and OSF 
Preprints [osf.io/preprints/] offer a good way of making the pre-print available for citation before actual publication happens.

While journal's strict demands on formatting, and delays in peer-review remain the points of annoyance, too much emphasis on Impact Factor (IF) is another such issue. Is not 'Impact' more important than IF? DORA declaration (San Francisco Declaration on Research Assessment) talks about several valid points in this context [http://www.ascb.org/dora/]. In general, there is a need for better ways to be adopted for assessment of research and researchers. Over-emphasis on journal metrics like IF has tempted some publishers to adopt dubious ways to artificially enhance scores of their journals. Evaluating an individual and their contribution is more important for decisions like promotion and awarding research grant, than evaluating journals. Citation count and h-index type of scores actually provide a more direct measure of scientific contribution made by an individual scientist or institute [https://indiabioscience.org/columns/opinion/a-new-yardstick-is-citation-count-a-morerealistic-measure-of-research-impact].

In summary, it is recommended that reducing the emphasis on formatting requirements and IF of journals, along with expedited peer-review will do an overall good to the scientific community. This will allow the researchers to have more quality time to spend on their lab bench, and being more productive. 